The following rules have been agreed upon between the Editors of the Fournal of the Chemical Society and of the Biochemical Fournal, after consultation with their American colleagues, and are accepted by the Editors of the British Fournal of Nutrition. The examples are given solely to illustrate the application of the rules; in other respects (e.g. the method of enumeration of carbon atoms) names will naturally follow the customary usages of the journal in which they are to appear.

Rule I. In cases where no possibility of misunderstanding can arise regarding which isomer of the aminoacid is under discussion, the amino-acid may be named without prefix or symbol to denote the direction of the rotation.

RULE 2. In cases where distinction between the stereoisomers is necessary, or considered desirable for the sake of clarity, the configurational family to which the alpha carbon atom belongs should be designated by a prefixed small capital letter. An additional sign to denote the direction of the rotation is not necessary.

\section{Examples: L-leucine, D-valine.}

The optically inactive mixture or racemic compound should, except where Rule I applies, be designated with the prefix DL.

\section{Example: DL-leucine.}

RuLE 3. The configuration of carbohydrates and other compounds which can be configurationally related to glyceraldehyde is designated by a prefixed $D$ or $L$. Where confusion is possible between the use of the small capital letter prefix for the configuration of the alpha carbon atom in amino-acid nomenclature and for that of the highest numbered asymmetric carbon atom in carbohydrate nomenclature, a subscript is added to the small capital letter prefix. Where the prefix is used in the amino-acid sense, the subscript ' $s$ ' is added; where the prefix is used in the carbohydrate sense, the subscript ' $g$ ' is added. These subscripts refer, respectively, to serine, the fundamental substance to which amino-acids that bear structural resemblance to the carbohydrates can be formally related, and to glyceraldehyde, the fundamental substance to which the configuration of the carbohydrates is formally related.

Examples: L,-threonine, for which the synonym in carbohydrate nomenclature is 2-amino-2:4-dideoxy- $D_{g}$ threonic acid; $D_{s}$-threonine, for which the synonym is 2-amino-2:4-dideoxy- $\mathrm{L}_{\boldsymbol{g}}$-threonic acid; $\mathrm{L}_{\mathrm{g}}$-allothreonine, for which the synonym is 2-amino-2:4-dideoxy- $\mathrm{L}_{\mathrm{g}}$-erythronic acid; $D_{s}$-allothreonine, for which the synonym is 2-amino-2:4-dideoxy- $D_{g}$-erythronic acid.
Papers that deal with both carbohydrates and aminoacids and which make use of the prefixes should employ subscripts at least for the names of the amino-acids. The acids derived from amino sugars should be named in conformity with carbohydrate nomenclature but with the use of the subscript: e.g. $D_{g}$-glucosaminic acid for 2-amino-2-deoxy-Do-gluconic acid.

RuLE 4. If the configurational relationship of the alpha carbon atom has not been definitely established, the actual direction of the rotation in a specified solvent, preferably of the free amino-acid in water, is designated by the prefixes dextro or laevo or, alternatively, by a plus or a minus sign enclosed in parenthesis.

Examples: dextrohydroxytryptophan. (+)-hydroxytryptophan.

Rule 5. The prefix meso should be used for the meso isomers of amino-acids and their derivatives that are optically inactive because of internal compensation.

\section{Examples: mesolanthionine, mesocystine.}

RuLE 6. Where the amino-acid has two asymmetric centres, but where internal compensation is impossible, the form which occurs in proteins should be called the L-amino-acid, and the form which has opposite configuration on both asymmetric carbon atoms should be designated the D-amino-acid. The pair having opposite configurations on one asymmetric centre only should be designated $\mathrm{D}$-allo and $\mathrm{L}$-allo, $\mathrm{D}$ and $\mathrm{L}$ referring as usual to the alpha carbon atom.

\section{Examples: D-allothreonine, L-allohydroxyproline.}

RULE 7. Derivatives and salts of amino-acids and peptides should, where necessary, be named with the use of D or $L$ to denote the configurational family of the alpha carbon atom or atoms, the customary rules being otherwise observed.

Examples: acetyl-L-tryptophan, L-histidine monohydrochloride monohydrate, copper L-aspartate, Dleucyl-D-valine.

RULE 8. Where a trivial name is applied to a compound that contains two alpha amino carboxylic acid groupings attached to dissimilar carbon chains, the configurational designation is that of the alpha carbon atom of the smaller of the two chains.

Examples: D-cystathionine for $S$-(D- $\beta$-amino- $\beta$-carboxyethyl)-D-homocysteine, L-allocystathionine for $S-(\mathrm{L}-$ $\beta$-amino- $\beta$-carboxyethyl)-D-homocysteine.

It is emphasized that the introduction of new trivial names should be avoided. 